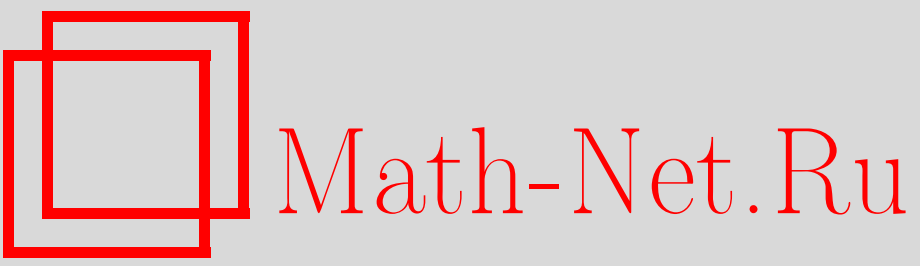

А. Я. Казаков, Начальная задача и усреднение в фоковском пространстве, ТMФ, 1998, том 117, номер 1, 92-106

DOI: https://doi.org/10.4213/tmf919

Использование Общероссийского математического портала Math-Net.Ru подразумевает, что вы прочитали и согласны с пользовательским соглашением

http://www.mathnet.ru/rus/agreement

Параметры загрузки:

IP : 3.85 .7 .115

26 апреля 2023 г., 12:29:12 
ТЕОРЕТИЧЕСКАЯ

И МАТЕМАТИЧЕСКАЯ

ФИЗИКА

Том 117, № 1

октябрь, 1998

(C) 1998 г.

А.Я. Казаков*

\title{
НАЧАЛЬНАЯ ЗАДАЧА И УСРЕДНЕНИЕ В ФОКОВСКОМ ПРОСТРАНСТВЕ
}

\begin{abstract}
Обсуждается динамика двухуровневого атома, одновременно взаимодействующего с классическим и квантованным полями. Изучается соответствующая начальная задача в фоковском пространстве для зависящего от времени гамильтониана. Получена оценка решения для специального класса начальных данных. Показано, что рассматриваемый класс начальных состояний сохраняется при эволюции системы. Предложен метод усреднения для построения решения начальной задачи в случае, если гамильтониан зависит от частот, резко отличающихся по порядку величины. Получена оценка поправки к найденному методом усреднения решению начальной задачи.
\end{abstract}

\section{1. ВВЕДЕНИЕ}

В данной работе мы обсудим динамику атома, взаимодействуюшего одновременно с квантованным и классическим полями. При этом нас интересует “квантово-оптическая" ситуация, т.е. мы предполагаем, что внешнее поле квазирезонансно одному или нескольким атомным переходам. Такие физические системы в последние годы активно обсуждаются в квантовой оптике в связи с возможными приложениями при создании микромазеров и проведении прецизионных экспериментов. Для этих задач динамику системы следует описьвать с помощью гамильтониана, явно зависящего от времени. В таких задачах сушествуют естественные частотные параметры, сильно отличающиеся по порядку величины. Оптическая частота (частота атомного перехода, а также близкие к ней частота квантованного поля и несущая частота классического поля) принимает значения порядка $10^{15}$ Гц и значительно (на 6 и более порядков) превосходит другие частотные параметры. С амплитудой внешнего поля можно связать так называемый параметр Раби (частоту Раби), эффективно описывающий взаимодействие поля с атомом. Для классического поля эта величина принимает значения вплоть до $10^{8}$ Гц. Для квантованного поля соответствующий параметр Раби сушественно меньше и принимает значения вплоть до $10^{3}-10^{4}$ Гц. Еще одним частотным параметром является частота модуляции внешнего классического поля, которая реально может принимать произвольные значения в диапазоне $0-10^{8}$ Гц.

* Государственный университет аэрокосмического приборостроения, Санкт-Петербург, Россия. E-mail: akaz@phsc2.stu.neva.ru 
Таким образом, возникает необходимость в построении подходящего асимпотического аппарата, позволяющего последовательно вычислять необходимые физические величины с учетом естественной иерархии масштабов частот. Данная работа посвяшена этой задаче в частном, но важном для приложений случае однофотонного взаимодействия двухуровневого атома с внешним полем. С точки зрения обычной теории возмушений наибольшая сложность связана с наличием в поправочных членах неограниченных операторов и их степеней. В связи с этим необходимо на первом этапе получить оценку специального вида для решения соответствующей начальной задачи и затем использовать эту информацию для оценки погрешности асимптотической процедуры.

Еше одна особенность рассматриваемого круга задач связана с тем, что решение начальной задачи сушествует не для произвольных начальных данных. Эта особенность характерна для моделей с неограниченными операторами [1]. Поэтому приходится ограничиваться рассмотрением выделенного класса начальных состояний. В нашем случае, однако, этот класс включает в себя все физически интересные начальные состояния, обычно используемые в приложениях.

Во втором разделе данной статьи описана постановка задачи. В разделе 3 доказана теорема об оценке решения начальной задачи. В разделе 4 описан соответствуюший вариант теории возмущений (процедура усреднения), позволяющий последовательно строить старший асимптотический член физической задачи и оценивать поправочный член. В разделе 5 процедура усреднения рассмотрена в случае классического монохроматического поля. Некоторые детали вычислений вынесены в приложение.

\section{2. ВЗАИМОДЕЙСТВИЕ ДВУХУРОВНЕВОГО АТОМА С КВАНТОВАННЫМ И КЛАССИЧЕСКИМ ПОЛЯМИ}

Рассмотрим задачу о двухуровневом атоме, взаимодействуюшем одновременно с квазирезонансными классическим и квантованным полями. Обсуждать эту задачу будем в рамках надлежашим образом модифицированной модели Джейнса-Каммингса.

Исходным является гамильтониан

$$
\begin{aligned}
\mathbf{H}= & \omega \mathbf{a}^{+} \mathbf{a}+\kappa \mathbf{J}_{0}+\zeta\left[\left(\mathbf{a}^{+}+\mathbf{a}\right) \mathbf{J}_{-}+\left(\mathbf{a}^{+}+\mathbf{a}\right) \mathbf{J}_{+}\right]+ \\
& +\mu\left[\mathbf{J}_{-} \int_{D} d \delta b(\delta) \cos [(\Omega+\delta) t]+\mathbf{J}_{+} \int_{D} d \delta b(\delta) \cos [(\Omega+\delta) t]\right] .
\end{aligned}
$$

Операторы

$$
\mathbf{J}_{0}=\left(\begin{array}{cc}
1 & 0 \\
0 & -1
\end{array}\right), \quad \mathbf{J}_{-}=\mathbf{J}_{+}^{T}=\left(\begin{array}{ll}
0 & 0 \\
1 & 0
\end{array}\right)
$$

описывают двухуровневый атом и его взаимодействие с квазирезонансным внешним полем. Они удовлетворяют коммутационным соотношениям

$$
\left[\mathbf{J}_{0}, \mathbf{J}_{-}\right]=-2 \mathbf{J}_{-}, \quad\left[\mathbf{J}_{0}, \mathbf{J}_{+}\right]=2 \mathbf{J}_{+} \cdot
$$

В формуле (1) $\omega$-частота квантованной моды внешнего поля, $\Omega$ - несушая (оптическая) частота внешнего классического поля, $b(\delta)$ - модуляция внешнего поля, константа $\mu(\zeta)$ 
задает взаимодействие классического ( квантованного) поля с атомным переходом. В члене, описывающем взаимодействие с классическим полем, параметр $\mu$ фиксируется условием

$$
\int_{D}|b(\delta)| d \delta=1
$$

Гамильтониан (1) действует в пространстве $\mathbf{G}=\mathbf{R}^{2} \otimes \mathbf{F}$, где $\mathbf{F}$ - фоковское пространство, в котором заданы операторы рождения и уничтожения $\mathbf{a}, \mathbf{a}^{+} ;\left[\mathbf{a}, \mathbf{a}^{+}\right]=1$.

Член $\omega \mathbf{a}^{+} \mathbf{a}$ соответствует гамильтониану свободного квантованного поля, член $\kappa \mathbf{J}_{0}-$ гамильтониану двухуровневого атома. В качестве базиса в пространстве $\mathbf{F}$ используются состояния $|n\rangle=\left(\mathbf{a}^{+}\right)^{n}|0\rangle$, где $|0\rangle$ - вакуум. Эти состояния ненормированы, так что $\langle n \mid n\rangle=n$ !. Имеют место соотношения

$$
\mathbf{a}^{+}|n\rangle=|n+1\rangle, \quad \mathbf{a}|n\rangle=n|n-1\rangle .
$$

В качестве норм векторов и операторов в $\mathbf{R}^{2}$ мы используем обычные евклидовы нормы, а в качестве нормы в пространстве $\mathbf{G}$ для состояний

$$
\Phi=\sum_{n \geqslant 0} \Phi_{n}|n\rangle,
$$

где $\Phi_{n}$ - двумерные векторы, можно использовать норму

$$
\|\Phi\|_{\mathrm{G}}^{2}=\sum_{n \geqslant 0} n !\left\|\Phi_{n}\right\|^{2} .
$$

Гамильтониан (1) написан вне рамок так называемого приближения вращающейся волны. Подобные гамильтонианы использовались при анализе ряда физических задач в случае монохроматического классического поля при дополнительных условиях типа $\omega=\Omega$ или $\omega=2 \kappa=\Omega$ в работах [2-4].

Нас интересует динамика волновой функции, являюшейся решением уравнения Шредингера

$$
i \frac{\partial}{\partial t} \Psi=\mathbf{H} \Psi
$$

при некоторых начальных данных (их выбор мы обсудим позднее). Как упоминалось выше, в этой задаче имеется несколько частотных параметров, сильно различаюшихся по порядку величины. Параметр Раби классического поля $R$ (можно считать его равным параметру $\mu$ в гамильтониане (1)) и тем более параметр Раби квантованной моды $R_{\mathrm{q}}$ $\left(R_{\mathrm{q}}=\zeta \sqrt{N}\right.$, где $N$ - число фотонов в квантованной моде) значительно меньше оптических частот $\omega, \Omega$. Пусть $D=\left[-D_{1}, D_{2}\right], D_{3}=\left|D_{2}-D_{1}\right|, \Delta=\Omega-\omega$. Параметры $D_{1}$ и $D_{2}$ описывают границы спектра модуляции классического внешнего поля, параметр $D_{3}$ - его ширину. Мы предполагаем, что параметры $\Delta$ и $D_{3}$ имеют тот же порядок величины, что и $\mu$.

Условие квазирезонансности означает, что $|2 \kappa-\omega| \sim|2 \kappa-\Omega| \sim \mu$. При анализе физических задач обычно опускают в гамильтониане "нерезонансные" члены, в том числе 
члены типа $\mathbf{a}^{+} \mathbf{J}_{-}, \mathbf{a} \mathbf{J}_{+}$, оставляя "резонансные" члены типа $\mathbf{a}^{+} \mathbf{J}_{+}, \mathbf{a J}_{-}$. В этом, по существу, и заключается приближение вращающейся волны, которое эквивалентно некоторой процедуре усреднения. Однако такое упрошение означает отбрасывание членов, включаюших неограниченные операторы, и нуждается в дополнительном обосновании. Обсуждаемая в этой статье задача и состоит в обосновании приближения, аналогичного обычному приближению вращающейся волны, в случае, когда атом одновременно взаимодействует с квантованным и модулированным классическим полями.

Сделаем одно замечание. Часто процедура усреднения приводит к гамильтониану с интегралом движения. Такая ситуация реализуется, например, в случае модели Джейнса-Каммингса, когда после отбрасывания членов с оптическими частотами получается гамильтониан, сохраняющий число возбуждений в системе,

$$
\mathbf{H}_{\mathrm{JC}}=\omega \mathbf{a}^{+} \mathbf{a}+\kappa \mathbf{J}_{0}+\zeta\left[\left(\mathbf{a}^{+}+\mathbf{a}\right) \mathbf{J}_{-}+\left(\mathbf{a}^{+}+\mathbf{a}\right) \mathbf{J}_{+}\right] .
$$

При этом процедура усреднения и получение соответствующей оценки существенно упрошаются, т.к. задача сводится к набору конечномерных задач. Однако при взаимодействии атома одновременно с классическим и квантованным полями этот интеграл движения отсутствует. Анализу именно этой ситуации посвяшена данная работа.

Приведем гамильтониан (1) к виду, допускаюшему применение асимптотической процедуры. Совершим преобразование

$$
\Psi(t)=\exp \left[-i \omega t\left(\mathbf{a}^{+} \mathbf{a}+\frac{\mathbf{J}_{0}}{2}\right)\right] \Phi(t) .
$$

Используя коммутационные соотношения

$$
\begin{gathered}
f\left(\mathbf{a}^{+} \mathbf{a}+\frac{\mathbf{J}_{0}}{2}\right) \mathbf{a}^{+} \mathbf{J}_{+}=\mathbf{a}^{+} \mathbf{J}_{+} f\left(\mathbf{a}^{+} \mathbf{a}+\frac{\mathbf{J}_{0}}{2}+2\right), \\
f\left(\mathbf{a}^{+} \mathbf{a}+\frac{\mathbf{J}_{0}}{2}\right) \mathbf{a} \mathbf{J}_{-}=\mathbf{a} \mathbf{J}_{-} f\left(\mathbf{a}^{+} \mathbf{a}+\frac{\mathbf{J}_{0}}{2}-2\right),
\end{gathered}
$$

находим уравнение для функции $\Phi(t)$

$$
i \frac{\partial \Phi(t)}{\partial t}=\left(\mathbf{H}_{\mathrm{q}}+\mathbf{H}_{\mathrm{cl}}\right) \Phi(t),
$$

где

$$
\begin{aligned}
\mathbf{H}_{\mathrm{q}}= & \zeta\left[\mathbf{a}^{+} \mathbf{J}_{-}+\mathbf{a} \mathbf{J}_{+}+\mathbf{a}^{+} \mathbf{J}_{+} \exp (2 i \omega t)+\mathbf{a} \mathbf{J}_{-} \exp (-2 i \omega t)\right], \\
\mathbf{H}_{\mathrm{cl}}= & \mu\left[\mathbf{J}_{-} B(t) \exp (i \Delta t)+\mathbf{J}_{+} \overline{B(t)} \exp (-i \Delta t)\right]+\left(\kappa-\frac{\omega}{2}\right) \mathbf{J}_{0}+ \\
& +\mu\left[\mathbf{J}_{-} \overline{B(t)} \exp (-i(\omega+\Omega) t)+\mathbf{J}_{+} B(t) \exp (i(\omega+\Omega) t)\right], \\
B(t)= & \int_{D} d \delta b(\delta) \exp [i \delta t] .
\end{aligned}
$$


Здесь $\mathbf{H}_{\mathrm{q}}$ и $\mathbf{H}_{\mathrm{cl}}$ - соответственно квантовая и классическая части приведенного гамильтониана.

Нам потребуется решение следуюшей вспомогательной задачи:

$$
i \frac{\partial M(t)}{\partial t}=\mathbf{H}_{\mathrm{cl}} M(t), \quad M(0)=\left(\begin{array}{l}
1 \\
0
\end{array}\right) .
$$

Положим

$$
M(t)=\left(\begin{array}{l}
m_{2}(t) \\
m_{1}(t)
\end{array}\right)
$$

Нетрудно проверить, что

$$
\left|m_{1}(t)\right|^{2}+\left|m_{2}(t)\right|^{2}=1 \text {. }
$$

Рассмотрим решение начальной задачи

$$
i \frac{\partial \Xi(t)}{\partial t}=\mathbf{H}_{\mathrm{cl}} \Xi(t), \quad \Xi(0)=\left(\begin{array}{ll}
1 & 0 \\
0 & 1
\end{array}\right)
$$

Матричную функцию можно записать в виде

$$
\Xi(t)=\left(\begin{array}{cc}
m_{2}(t) & -\overline{m_{1}(t)} \\
m_{1}(t) & \overline{m_{2}(t)}
\end{array}\right),
$$

причем, как следует из (8), она обладает свойством $\|\Xi(t)\|=1$.

Будем искать решение уравнения (6) в виде $\Phi(t)=\Xi(t) \Theta(t)$. Функция $\Theta(t)$ удовлетворяет уравнению

$$
i \frac{\partial \Theta(t)}{\partial t}=\Xi^{-1}(t) \mathbf{H}_{\mathrm{q}} \Xi(t) \Theta(t)
$$

где

$$
\begin{aligned}
\Xi^{-1}(t) \mathbf{H}_{\mathrm{q}} \Xi(t)= & \zeta\left(\begin{array}{cc}
\overline{m_{1}} m_{2} & -\overline{m_{1}^{2}} \\
m_{2}^{2} & -\overline{m_{1}} m_{2}
\end{array}\right)\left[\mathbf{a}^{+}+\mathbf{a} \exp (-2 i \omega t)\right]+ \\
& +\zeta\left(\begin{array}{cc}
\overline{m_{2}} m_{1} & \overline{m_{2}^{2}} \\
-m_{1}^{2} & -\overline{m_{2}} m_{1}
\end{array}\right)\left[\mathbf{a}+\mathbf{a}^{+} \exp (2 i \omega t)\right] .
\end{aligned}
$$

Для введения безразмерного большого параметра сделаем замену переменной $\tau=\zeta t$. Как упоминалось выше, $\zeta$ - наименьший частотньй параметр задачи, так что отношение других частотных параметров (таких, как $\mu, \omega)$ к нему - большая величина. "Большими" мы считаем величины порядка параметра Раби классического поля, а "малыми" величины порядка параметра Раби квантованного поля. Таким образом, на физическом языке процедура усреднения - это отделение чисто квантовой, “медленной” эволюции системы от "быстрых" осцилляций, связанных со взаимодействием атома с классическим полем.

Запишем уравнение (9) в новых переменных в виде

$$
i \frac{\partial \Theta(\tau)}{\partial \tau}=P(\tau) \Theta(\tau) .
$$


При этом начальное значение сохраняется,

$$
\Theta(0)=\Psi_{0}
$$

Так как в нашем уравнении присутствуют “большие" частоты, то можно ввести понятие “быстрых" и “медленных" функций. Мы будем называть “быстрыми” (соответственно "медленными") такие функции, дифференцирование которых повышает (не изменяет) их асимптотический порядок. В соответствии с нашей терминологией можно записать, что

$$
P(\tau)=S(\tau)+F(\tau)
$$

При конкретном выборе коэффициентов в гамильтониане $\mathbf{H}_{\mathrm{cl}}$ нетрудно явно описать функции $S(\tau)$ и $F(\tau)$. Как следует из наших определений,

$$
P(\tau)=P_{-}(\tau) \mathbf{a}+P_{+}(\tau) \mathbf{a}^{+}
$$

для некоторых матричнозначных функций $P_{-}(\tau)$ и $P_{+}(\tau)$. Аналогичные обозначения можно ввести для $S(\tau)$ и $F(\tau)$.

Исходная начальная задача переписана теперь в форме, обычной для применения процедуры усреднения [5]. Однако в отличие от работы [5] “коэфффициент" в правой части (10) содержит неограниченные операторы.

\section{3. НАЧАЛЬНАЯ ЗАДАЧА}

Выведем оценку для решения следуюшей начальной задачи:

$$
\begin{aligned}
\frac{\partial \Psi(t)}{\partial t} & =\left[P_{-}(t) \mathbf{a}+P_{+}(t) \mathbf{a}^{+}\right] \Psi(t)+Q(t), \\
\Psi(0) & =\Psi_{0} .
\end{aligned}
$$

Здесь функции $Q(t)$ и $\Psi(t)$ принимают значения в пространстве $\mathbf{G}, P_{-}(t)$ и $P_{+}(t)$ - матричные операторы, действующие в $\mathbf{R}^{2}$, причем предполагается, что справедливы оценки

$$
\left\|P_{-}(t)\right\| \leqslant \frac{g}{2}, \quad\left\|P_{+}(t)\right\| \leqslant \frac{g}{2},
$$

где $g$ - некоторая постоянная. Мы будем рассматривать начальную задачу в несколько более общей постановке, чем задача $(10),(11)$, чтобы рассмотреть ее вариант, необходимый для исследования процедуры усреднения.

Решение задачи (13), (14) можно представить в виде

$$
\Psi(t)=\sum_{n \geqslant 0} \Psi_{n}(t)|n\rangle
$$

где $\Psi_{n}(t)$ - вектор-функция, принимающая значения в $\mathbf{R}^{2}$. Начальную задачу $(13),(14)$ перепишем в эквивалентном виде, проинтегрировав уравнение (13):

$$
\Psi(t)=\Psi_{0}+W(t)+\int_{0}^{t}\left[P_{-}(s) \mathbf{a}+P_{+}(s) \mathbf{a}^{+}\right] \Psi(s) d s,
$$


где

$$
W(t)=\int_{0}^{t} Q(s) d s
$$

Разложим начальное состояние и вектор-функцию $W(t)$ по состояниям $|n\rangle$ :

$$
\begin{aligned}
\Psi(0) & =\sum_{n \geqslant 0} \psi_{n}|n\rangle, \\
W(t) & =\sum_{n \geqslant 0} W_{n}(t)|n\rangle,
\end{aligned}
$$

причем $\psi_{n}, W_{n}(t)$ - векторы в $\mathbf{R}^{2}$. Аналогичным образом можно ввести и вектор-функции $Q_{n}(t)$.

ТЕОРЕМа 1. Пусть функиии $Q_{n}(t)$ непрерьвны при любом $t$ и справедливы оцен$\kappa u$

$$
\begin{aligned}
& \left\|\Psi_{n}^{(0)}\right\| \leqslant \frac{K \rho^{n}}{n !} \\
& \left\|W_{0,1}(t)\right\| \leqslant M, \\
& \left\|W_{n}(t)\right\| \leqslant M \frac{\lambda^{n}}{(n-2) !}, \quad n>1,
\end{aligned}
$$

где $\rho, \lambda \geqslant 1, \quad 0 \leqslant t \leqslant T$. Тогда решение начальной задачи (13), (14) существует при $0 \leqslant t \leqslant T$, причем

$$
\begin{aligned}
\left\|\Psi_{n}(t)\right\| \leqslant & \frac{K \rho^{n}}{n !}(1+2 \rho g t)^{n+1}\left[1+e^{4 \rho^{2} g^{2} t^{2}}\right]+ \\
& +\frac{4 M \lambda^{n}}{(n-2) !}(1+2 \lambda g t)^{n+1}\left[1+e^{4 \lambda^{2} g^{2} t^{2}}\right]
\end{aligned}
$$

npu $n \geqslant 2 u$

$$
\begin{aligned}
\left\|\Psi_{n}(t)\right\| \leqslant & \frac{K \rho^{n}}{n !}(1+2 \rho g t)^{n+1}\left[1+e^{4 \rho^{2} g^{2} t^{2}}\right]+ \\
& +4 M \lambda^{n}(1+2 \lambda g t)^{n+1}\left[1+e^{4 \lambda^{2} g^{2} t^{2}}\right]
\end{aligned}
$$

при $n=0,1$.

ДокАЗАтЕльство. Решая уравнение (17) с помощью итераций, получаем, что

$$
\Psi(t)=\sum_{k \geqslant 0} \Psi^{(k)}(t),
$$

где

$$
\Psi^{(0)}(t)=\Psi_{0}+W(t)
$$

и

$$
\Psi^{(k+1)}(t)=\int_{0}^{t}\left[P_{-}(x) \mathbf{a}+P_{+}(x) \mathbf{a}^{+}\right] \Psi^{(k)}(x) d x
$$


Из соотношения (23) следует, что

$$
\begin{aligned}
\Psi^{(k)}(t)= & \int_{0}^{t} \cdots \int_{0}^{x_{2}}\left[P_{-}\left(x_{k}\right) \mathbf{a}+P_{+}\left(x_{k}\right) \mathbf{a}^{+}\right] \times \cdots \\
& \cdots \times\left[P_{-}\left(x_{1}\right) \mathbf{a}+P_{+}\left(x_{1}\right) \mathbf{a}^{+}\right]\left(\Psi_{0}+W\left(x_{1}\right)\right) d x_{k} \ldots d x_{1} .
\end{aligned}
$$

Разложим вектор-функции $\Psi^{(k)}(t)$ по состояниям чисел заполнения,

$$
\Psi^{(k)}(t)=\sum_{n \geqslant 0} \Psi_{n}^{(k)}(t)|n\rangle,
$$

при этом

$$
\Psi_{n}(t)=\sum_{k \geqslant 0} \Psi_{n}^{(k)}(t)
$$

Мы будем оценивать величину $\left\|\Psi_{n}^{(k)}(t)\right\|$ для всех $k, n$, а затем с помошью соотношения $(25)$ получим оценку для $\Psi_{n}(t)$. В силу линейности начальной задачи достаточно по отдельности обсудить ситуации, когда $Q(t)=0$ и $\Psi_{0}=0$. Анализ обоих вариантов, по существу, одинаков, мы будем полагать $Q(t)=0$.

Рассмотрим случай $k \leqslant n$. Величина $\Psi_{n}^{(k)}(t)$ в правой части $(24)$ порождается $2^{k}$ слагаемыми, каждое из которых возникает при $k$-кратном интегрировании $\Psi_{l}^{(0)}$ для некоторого $l . k$-Кратное интегрирование приводит к множителю $(k !)^{-1}$, а действие операторов $P_{-}(t)$ и $P_{+}(t)$ можно оценить с помощью константы $(g / 2)^{k}$. Таким образом (детали приведены в приложении), мы приходим к следующему результату:

$$
\left\|\Psi_{n}^{(k)}(t)\right\| \leqslant K \frac{(2 \rho g t)^{k} \rho^{n}}{k !(n-k) !}
$$

Обсудим теперь случай $k>n$. Используя соотношение (23) и индукцию по $k$, получаем (детали см. в приложении), что

$$
\left\|\Psi_{n}^{(k)}(t)\right\| \leqslant K \frac{(2 \rho g t)^{k} \rho^{n}}{n !\left[\frac{k-n}{2}\right] !} .
$$

Применяя неравенство

$$
\left\|\Psi_{n}(t)\right\| \leqslant \sum_{k=0}^{n}\left\|\Psi_{n}^{(k)}(t)\right\|+\sum_{k>n}\left\|\Psi_{n}^{(k)}(t)\right\|
$$

и суммируя по отдельности в первой и второй суммах (28) с учетом соотношений (26) и (27), мы приходим к тем слагаемым в оценках $(21),(22)$, которые включают константу $K$. Аналогичные рассмотрения приводят и ко второй части слагаемых в оценках (21), (22), включающих константу $M$.

Отметим некоторые особенности приведенного результата. Мы обсуждали ситуацию, когда взаимодействие атома с внешним полем однофотонное. Именно в этом случае можно применить прямую итерационную процедуру построения решения начальной

4 Теоретическая и математическая физика, т. 117, № 1, 1998 г. 
задачи и получить оценки для него. Если же в гамильтониане присутствуют члены, соответствуюшие многофотонному взаимодействию (например, $\mathbf{a}^{m}$ при $m \geqslant 2$ ), то наши методы дают вместо оценки (27) лишш оценку вида

$$
\left\|\Psi_{n}^{(k)}(t)\right\| \leqslant K \frac{(2 \rho g t)^{k} \rho^{n}\left(n+m\left[\frac{k-n}{2}\right]\right) !}{n ! k !} .
$$

Очевидно, что мы не можем с помошью неравенств $(28),(29)$ получить оценку для $\Psi_{n}(t)$.

Полученные выше результаты уточняют результаты работы [1]. Это связано с тем, что мы использовали при получении оценки решения специфику рассматриваемого гамильтониана. Если сравнить оценки (19) и (21), то теорему можно переформулировать как утверждение об устойчивости класса состояний (19) под воздействием динамики (13). Класс состояний (19) включает в себя когерентные состояния [6] и состояния с конечным числом фотонов.

\section{4. ПРОЦЕДУРА УСРЕДНЕНИЯ В ФОКОВСКОМ ПРОСТРАНСТВЕ}

Применим полученные выше результаты для начальной задачи (10), (11). Обсудим здесь процедуру усреднения, которую необходимо использовать для построения старшего члена асимптотического решения этой задачи. Будем считать, что $0 \leqslant \tau \leqslant T$, $T \sim O(1)$. В исходном временном масштабе это означает, что решение рассматривается вплоть до времен $t=T / \zeta \gg 1$.

Будем считать, что “быстрые" гармоники осциллируют с частотами порядка $N$, где $N=\mu / \zeta \gg 1$. Положим

$$
\int_{0}^{\tau} d x F(x)=N^{-1} G(\tau)
$$

где $G(\tau)=G_{+}(\tau) \mathbf{a}^{+}+G_{-}(\tau) \mathbf{a}$. Аналогичное разложение будем использовать и для $S(\tau)$.

Пусть выполняются следующие условия:

1) для начального вектора $\Psi_{0}$ справедливы неравенства (19);

$2)$ функции $G_{+}(\tau), G_{-}(\tau), S_{+}(\tau)$ и $S_{-}(\tau)$ непрерывны при всех $\tau$, причем для $\alpha= \pm$, $0 \leqslant \tau \leqslant T, T=O(1)$ имеют место оценки $\left\|G_{\alpha}(\tau)\right\| \leqslant q / 2,\left\|S_{\alpha}(\tau)\right\| \leqslant s / 2$.

Рассмотрим начальную задачу

$$
\begin{aligned}
\frac{d \Theta_{0}(\tau)}{d \tau} & =S(\tau) \Theta_{0}(\tau), \\
\Phi_{0}(0) & =\Psi_{0} .
\end{aligned}
$$

Функция $\Theta_{0}(\tau)$ - это старший член асимптотического решения начальной задачи (10), (11), и уравнение для нее получается усреднением уравнения (10). Из условий 1 и 2 и теоремы 1 следует, что начальная задача (30), (31) имеет решение при всех $\tau$ и вьполнимы неравенства

$$
\left\|\left[\Theta_{0}(\tau)\right]_{n}\right\| \leqslant M \frac{\Lambda^{n}}{n !}, \quad n \geqslant 0,
$$


где

$$
M=K\left[1+\exp \left(4 \rho^{2} g^{2} t^{2}\right)\right], \quad \Lambda=\rho(1+2 \rho g t) .
$$

Для поправки к решению усредненной задачи $\Theta_{1}(\tau)=\Theta(\tau)-\Theta_{0}(\tau)$ получаем следующую начальную задачу:

$$
\begin{aligned}
\frac{d \Theta_{1}(\tau)}{d \tau} & =F(\tau) \Theta_{0}(\tau)+M(\tau) \Theta_{1}(\tau) . \\
\Theta_{1}(0) & =0 .
\end{aligned}
$$

Задачу (33), (34) можно переписать в виде

$$
\Theta_{1}(\tau)=\int_{0}^{\tau} d x F(x) \Theta_{0}(x)+\int_{0}^{\tau} d x M(x) \Theta_{1}(x) .
$$

Преобразуем неоднородный член в правой части (35), интегрируя по частям,

$$
\Theta_{1}(\tau)=L(\tau)+\int_{0}^{\tau} d x M(x) \Theta_{1}(x),
$$

где

$$
L(\tau)=N^{-1} G(\tau) \Theta_{0}(\tau)-N^{-1} \int_{0}^{\tau} d x G(x) S(x) \Theta_{0}(x) .
$$

Здесь было использовано уравнение (30). Из оценки (32) и условия 2 получаем

$$
\begin{aligned}
& \left\|\left[G(\tau) \Theta_{0}(\tau)\right]_{0}\right\| \leqslant M q, \\
& \left\|\left[G(\tau) \Theta_{0}(\tau)\right]_{n}\right\| \leqslant M q \frac{\Lambda^{n}}{(n-1) !}, \quad n \geqslant 1 .
\end{aligned}
$$

Аналогичным образом находим

$$
\begin{array}{ll}
\left\|\left[\int_{0}^{\tau} G(x) S(x) \Theta_{0}(x) d x\right]_{n}\right\| \leqslant M q s \frac{\Lambda^{n} T}{(n-2) !}, & n \geqslant 2, \\
\left\|\left[\int_{0}^{\tau} G(x) S(x) \Theta_{0}(x) d x\right]_{n}\right\| \leqslant M q s \Lambda^{n} T, \quad n=0,1 .
\end{array}
$$

Таким образом, для неоднородного члена в уравнении (36) можно записать следуюшие неравенства:

$$
\begin{array}{ll}
\left\|[L(\tau)]_{n}\right\| \leqslant N^{-1} M q(1+s)(T+1) \frac{\Lambda^{n}}{(n-2) !}, & n \geqslant 2, \\
\left\|[L(\tau)]_{n}\right\| \leqslant N^{-1} M q(1+s)(T+1) \Lambda^{n}, & n=0,1 .
\end{array}
$$

Вновь используя теорему 1 , но уже для уравнения (36), приходим к оценкам

$$
\begin{array}{lll}
\left\|\left[\Theta_{1}(\tau)\right]_{n}\right\| \leqslant N^{-1} M q(1+s)(T+1) \frac{\Lambda^{n}}{(n-2) !} F_{n}(\tau), & & n \geqslant 2, \\
\left\|\left[\Theta_{1}(\tau)\right]_{n}\right\| \leqslant N^{-1} M q(1+s)(T+1) \Lambda^{n} F_{n}(\tau), & & n=0,1,
\end{array}
$$

где

$$
F_{n}(\tau)=(1+2 \lambda g t)^{n+1}\left[1+e^{4 \Lambda^{2} g^{2} t^{2}}\right] .
$$

Резюмируем эти результаты. 
ТеОрема 2. Пусть справедливы условия 1 и 2. Тогда решение начальной задачи (10), (11) можно представить в виде $\Theta(\tau)=\Theta_{0}(\tau)+\Theta_{1}(\tau)$, причем функиия $\Theta_{0}(\tau)$ является решением усредненной задачи (30), (31), а функция $\Theta_{1}(\tau)$ удовлетворяет оченкам (37), (38).

Разумеется, из этих оценок следует соответствуюшая оценка для функции $\Theta_{1}(\tau)$ в норме пространства $\mathbf{G}$.

Итак, мы описали процедуру построения старшего члена асимптотического решения начальной задачи $(10),(11)$ и получили оценку поправки. Отметим, что для нормы $\Theta_{1}(t)$ получена оценка, растущая при увеличении $t$ как $1+\exp \left\{(2 \rho g t)^{4}\right\}$. Эта оценка слишком груба. Тем не менее из наших результатов следует, что эта оценка линейна по малому параметру $N^{-1}$. Таким образом, наша процедура усреднения действительно дает главный член асимптотического решения.

Справедливость условия 2 следует проверять для каждого конкретного выбора функции $B(t)$, описывающей модуляцию внешнего классического поля. Рассмотрим пример полихроматического внешнего классического поля. В этом случае оно представляет собой набор эквидистантных гармоник, т.е.

$$
B(t)=\sum_{n} b_{n} e^{i \delta n t}
$$

При этом предполагается, что $\delta \sim \mu$, коэффициенты $b_{n}=O(1)$ в соответствии с нашим выбором нормировки параметра $\mu$. Для того чтобы построить в старшем приближении по параметру $\Omega / \zeta \gg 1$ решение начальной задачи $(10),(11)$, достаточно учесть лишь "резонансные" члены в $\mathbf{H}_{\mathrm{cl}}$, отбрасывая осциллируюшие с оптическими частотами $(\Omega+\omega)$ члены (это следствие обычной теории усреднения [5], примененной к задаче $(7))$. Оставляя лиш резонансные члены, мы приходим к системе уравнений, которая с точностью до элементарного преобразования совпадает с системой с периодическими коэффициентами. Используя теорему Флоке, получаем следующее представление компонент вектор-функции:

$$
\begin{aligned}
& m_{1}(t)=e^{i(-\Delta+\nu) t} s_{1}(\delta t) \\
& m_{2}(t)=e^{i(\Delta+\nu) t} s_{2}(\delta t)
\end{aligned}
$$

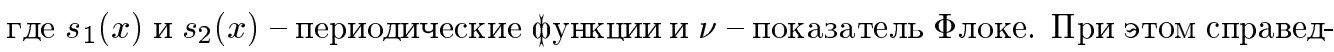
ливо соотношение

$$
\left|s_{1}(x)\right|^{2}+\left|s_{2}(x)\right|^{2}=1 .
$$

Рассмотрим в качестве примера функцию $\left[P_{+}(\tau)\right]_{1,1}$. Нетрудно вывести для нее явное выражение

$$
\left[P_{+}(\tau)\right]_{1,1}=e^{2 i \Delta \tau / \zeta} s_{2}\left(\frac{\delta \tau}{\zeta}\right) \overline{s_{1}(\delta \tau / \zeta)}
$$

Используя периодичность функций $s_{1}(x)$ и $s_{2}(x)$, получаем представление в виде ряда Фурье:

$$
\left[P_{+}(\tau)\right]_{1,1}=e^{2 i \Delta \tau / \zeta} \sum_{n} r_{n} e^{i n \delta \tau / \zeta}
$$


Для того чтобы построить функцию $S_{+}(\tau)$, в данном случае достаточно выделить из гармоник $(2 \Delta+n \delta) / \zeta$ ту, которая имеет величину порядка 1 (если таковая найдется, пусть ее номер будет $\left.n_{0}\right)$. Учитьвая соотношения $(39),(40)$, находим, что

$$
\left|\left[S_{+}(\tau)\right]_{1,1}\right|=\left|r_{n_{0}}\right| \leqslant 1 \text {. }
$$

Тогда для функции $F_{+}(\tau)$ (“быстрой” части функции $\left.P_{+}(\tau)\right)$ получаем

$$
\begin{aligned}
& \int_{0}^{\tau} d x\left[F_{+}(x)\right]_{1,1}=N^{-1} e^{2 i \Delta \tau / \zeta} \alpha(\tau), \\
& \alpha(\tau)=\widetilde{\sum_{n} \frac{r_{n} \mu e^{i n \delta \tau / \zeta}-1}{i(2 \Delta+\delta n)} .}
\end{aligned}
$$

Здесь суммирование идет по всем $n$, кроме $n=n_{0}$ (т.е. по быстрым гармоникам). Функция $\alpha(\tau)$ периодическая и непрерывная. Таким образом, она ограничена и выполняется условие 2.

\section{5. МОНОХРОМАТИЧЕСКОЕ КЛАССИЧЕСКОЕ ПОЛЕ}

Рассмотрим вопрос о том, какие гамильтонианы получаются при усреднении гамильтониана (9), на примере монохроматического классического поля. Если $g(t) \equiv 1$, то

$$
\Xi(t)=\exp \left[\frac{1}{2} i(\omega-\Omega) t \mathbf{J}_{0}\right] \mathbf{U} \exp \left[-i R t \mathbf{J}_{0}\right] \mathbf{U}^{-1},
$$

где

$$
\mathbf{U}=\left(\begin{array}{cc}
\mu & \Delta-R \\
R-\Delta & \mu
\end{array}\right), \quad R=\sqrt{\mu^{2}+\Delta^{2}}, \quad \Delta=\kappa-\frac{\Omega}{2} .
$$

Простые вычисления приводят к выводу, что оператор $\Xi(t)^{-1}\left(\mathbf{a}^{+} \mathbf{J}_{-}+\mathbf{a} \mathbf{J}_{+}\right) \Xi(t)$ содержит гармоники $\pm 2(\omega-\Omega), \pm 2(\omega-\Omega \pm R)$. Если они имеют тот же порядок, что и параметр Раби классического поля $R$, то усреднение исключит эти гармоники и они дадут вклад только в следуюшем асимптотическом порядке. Рассмотрим случай, когда одна из вышеупомянутых гармоник имеет частоту порядка $R_{\mathrm{q}} / R$. Тогда соответствуюший член войдет в усредненный гамильтониан. Ненулевой усредненный гамильтониан может появиться в следующих трех случаях:

1) $|R-\omega+\Omega|=|\sigma| \ll R$. При этом усредненный гамильтониан имеет вид

$$
\mathbf{H}_{1}=\zeta \mu^{2} D^{-1} \mathbf{U}\left(\begin{array}{cc}
0 & \mathbf{a} e^{2 i \sigma t} \\
\mathbf{a}^{+} e^{-2 i \sigma t} & 0
\end{array}\right) \mathbf{U}^{-1}
$$

где

$$
D=\operatorname{det} \mathbf{U}=\mu^{2}+(R-\Delta)^{2}
$$

Он отличается от стандартного гамильтониана модели Джейнса-Каммингса (после преобразования (5)) только поворотом; 
2) $|\omega-\Omega+R|=|\eta| \ll R$. В этом случае усредненный гамильтониан в правой части (9) имеет вид

$$
\mathbf{H}_{2}=-\zeta(R-\Delta)^{2} D^{-1} \mathbf{U}\left(\begin{array}{cc}
0 & \mathbf{a}^{+} e^{2 i \eta t} \\
\mathbf{a} e^{-2 i \eta t} & 0
\end{array}\right) \mathbf{U}^{-1}
$$

Как и ранее, этот гамильтониан сводится к гамильтониану модели Джейнса-Каммингса. В этой модели сушествует интеграл движения - число возбуждений. Для решения начальной задачи можно получить сушественно лучшую, чем оценки (21), (22), оценку, не содержащую экспоненциального роста со временем;

3) $|\omega-\Omega|=|\nu| \ll R$. Усреднение правой части (9) дает

$$
\mathbf{H}_{3}=\zeta \mu(R-\Delta) D^{-1}\left(\mathbf{a}^{+} e^{2 i \nu t}+\mathbf{a} e^{-2 i \nu t}\right) \mathbf{U} \mathbf{J}_{0} \mathbf{U}^{-1}
$$

Как следует из этого соотношения, система после двумерного поворота расщепляется на две идентичные подсистемы. Таким образом, после усреднения задача сводится к исследованию динамики с гамильтонианом

$$
\mathbf{H}_{4}=\rho\left[a^{+} e^{2 i \nu t}+a e^{-2 i \nu t}\right]
$$

где $\rho=\zeta \mu(R-\Delta) / D$, т.е. к решению начальной задачи для уравнения

$$
i \frac{\partial \varphi}{\partial t}=\rho\left[a^{+} e^{2 i \nu t}+a e^{-2 i \nu t}\right] \varphi
$$

В данном случае интеграла движения нет, число возбуждений не сохраняется. Соответствующую динамику удобно рассматривать в представлении Фока-Баргмана. Будем использовать представление $a^{+} \longmapsto z, a \longmapsto D_{z}$, где $D_{z}$ - оператор дифференцирования по $z$. Уравнение (42) принимает вид

$$
i \frac{\partial \varphi(z, t)}{\partial t}=\rho\left[z e^{2 i \nu t}+e^{-2 i \nu t} D_{z}\right] \varphi(z, t)
$$

После подстановки $z \exp (2 i \nu t)=y, t=\tau$ переменные в уравнении (43) разделяются, и его решение можно записать в виде

$$
\begin{aligned}
\varphi(z, t)= & \exp \left\{\frac{z \rho}{2 \nu}\left[1-e^{2 i \nu t}\right]+\right. \\
& \left.+\frac{\rho^{2}}{4 \nu^{2}}\left[e^{-2 i \nu t}+2 i \nu t-1\right]\right\} X\left[z+\frac{\rho}{2 \nu}\left(e^{-2 i \nu t}-1\right)\right]
\end{aligned}
$$

Функция $X(z)$ определяется из начальных условий. Если $\Phi(z, 0)=\Phi_{0}(z)$, то $X(z)=$ $\Phi_{0}(z)$. Среднее значение любой физической величины $G$ можно представить [6] как

$$
\langle G\rangle=\int \overline{\varphi(z, t)} G \varphi(z, t) e^{-z \bar{z}} d z d \bar{z}
$$

Физические следствия полученных соотношений мы обсудим в другом месте. 


\section{6. ЗАКЛЮЧЕНИЕ}

В статье в рамках модифицированной модели Джейнса-Каммингса рассмотрена динамика двухуровневого атома, взаимодействующего одновременно с классическим и квантованным полями. В этом случае гамильтониан системы включает гармоники сушественно разных порядков. Для подходящего класса начальных данных с помошью прямой итерационной процедуры получена оценка решения соответствуюшей начальной задачи. Построен метод усреднения, и получена оценка нормы поправки к решению усредненной задачи. Вывод аналогичных результатов в случае многофотонного взаимодействия атома с квантованным полем остается открытой проблемой.

ПРИЛОЖЕНИЕ

Докажем оценку

$$
\left\|\Psi_{n}^{(k)}(t)\right\| \leqslant K \frac{(2 \rho g t)^{k} \rho^{n}}{k !(n-k) !}
$$

где $k \leqslant n$. При доказательстве будем использовать соотношение $(24)$, раскрывая все скобки и подставляя разложение начального состояния по состояниям $|n\rangle$. Отдельные члены получившейся суммы (если представить это в виде диаграммы) удобно изображать ломаными на плоскости $(N, K)$. По оси $N$ при этом отмечается число фотонов $n$, по оси $K$ - порядок итерации $k$. Каждому слагаемому, входящему в $\Psi_{n}^{(k)}(t)$ (всего есть $2^{k}$ таких слагаемых), соответствует на этой диаграмме ломаная, которая начинается в точке $(n \pm r, 0), 0 \leqslant|r| \leqslant k$, и оканчивается в точке $(n, k)$. Каждое слагаемое содержит $k$ сомножителей типа $P_{-}(x), P_{+}(x)$, которые при оценке дают множитель $(g / 2)^{k} . k$-Кратное интегрирование порождает множитель $t^{k}(k !)^{-1}$. Очевидно, что действие оператора $\mathbf{a}^{+}$ не вносит дополнительных множителей (оператор $\mathbf{a}^{+}$только изменяет число фотонов n). Рассмотрим множители, связанные с действием оператора а. Максимальный вклад среди членов, соответствующих ломаным с одинаковыми началом и концом, как следует из соотношения (47), имеет член с ломаной, сначала идушей вверх вплоть до линии $N+K=k+n$, а затем вдоль этой линии - вниз до точки $(n, k)$. В первую очередь рассмотрим случай, когда начальная точка находится при $(n-r, 0), k \geqslant r>0$, а конечная - в точке $(n, k)$. Соответствуюшее слагаемое $T(n-r, 0 \Rightarrow n, k)$ может быть оценено следующим образом:

$$
\|T(n-r, 0 \Rightarrow n, k)\| \leqslant K \frac{\rho^{n-r}}{(n-r) !} \frac{(g t / 2)^{k}}{k !} \frac{(n+s) !}{n !} .
$$

Здесь $2 s+r=k$, и мы приняли во внимание соотношение (19). Последний сомножитель в (47) учитывает действие оператора а.

Используя аналогичные соображения, мы получаем оценку для слагаемых, ломаные которых начинаются в точке $(n+r, 0), k \geqslant r>0$. В этом случае

$$
\|T(n+r, 0 \Rightarrow n, k)\| \leqslant K \frac{\rho^{n+r}}{(n+r) !} \frac{(g t / 2)^{k}}{k !} \frac{(n+s+r) !}{n !},
$$


где $2 s+r=k$.

Докажем оценку

$$
\frac{(n+s) !}{(n-r) ! n !} \leqslant \frac{2^{k}}{(n-k) !}
$$

здесь $2 s+r=k, s, r \geqslant 0$. Неравенство (49) можно переписать в виде

$$
(n+s)(n+s-1) \ldots(n-r+1) \leqslant 2^{k} n(n-1) \ldots(n-k+1) .
$$

В левой части неравенства (50) содержится $s+r$ сомножителей, в правой части $n>s+r$ сомножителей, причем все они больше чем единица. Выбирая наибольшие $s+r$ сомножителей среди стоящих в правой части и умножая каждый из них на 2 , мы получаем сомножители типа $2(n-m), 0 \leqslant m \leqslant s+r$. Согласно нашим предположениям $2(n-m) \geqslant n+s-m(n \geqslant k=2 s+r)$. Это означает, что неравенство (50) и, следовательно, (49) справедливы.

Используя аналогичные соображения, мы получаем оценку

$$
\frac{(n+s+r) !}{(n+r) ! n !} \leqslant \frac{2^{k}}{(n-k) !}
$$

где $2 s+r=k, s, r \geqslant 0$. Функция $\Psi_{n}^{(k)}(t)$ является суммой $2^{k}$ слагаемых, причем каждое из них оценивается либо с помощью (49), либо с помощью (51). Используя (47), (49) и (48), (51), мы приходим к оценке (46).

Докажем теперь неравенство

$$
\left\|\Psi_{n}^{(k)}(t)\right\| \leqslant K \frac{(2 \rho g t)^{k} \rho^{n}}{n !\left(\left[\frac{k-n}{2}\right]\right) !}
$$

при $k>n$. Из соотношения (23) следует, что

$$
\Psi_{n}^{(k+1)}(t)=\int_{0}^{t} d x\left\{P_{-}(x) n \Psi_{n+1}^{(k)}(x)+P_{+}(x) \Psi_{n-1}^{(k)}(x)\right\} .
$$

Интегрирование по $x$ дает дополнительный множитель $(k+1)^{-1}$. Если оценка (52) справедлива для $\Psi_{n+1}^{(k)}, \Psi_{n-1}^{(k)}$, то с помошью последнего соотношения мы получаем оценку (52) для $\Psi_{n}^{(k+1)}(t)$. Для $k=n, k=n-1$ эта оценка вытекает из (46). Доказательство оценки завершается с помощью индукции.

\section{Список литературы}

[1] С. Г. Крейн. Дифференциальные уравнения в банаховом пространстве. М.: Наука, 1971.

[2] C. K. Law, J. H. Eberly. Phys. Rev. A. 1991. V. 43. P. 6337.

[3] P. Alsing, D.-S. Guo, H. J. Carmichael. Phys. Rev. A. 1992. V. 45. P. 5135.

[4] I. V. Jyotsna, G. S. Agarwal. Opt. Commun. 1993. V. 99. P. 344.

[5] A. H. Nayfeh. Introduction to Perturbation Techniques. N.Y.: J.Wiley and Sons, 1981.

[6] A. М. Переломов. Обобщенные когерентные состояния и их приложения. М.: Наука, 1987.

Поступила в редакцию 18.III.1998 г. 